\title{
Identification of a second gene associated with variation in vertebral number in domestic pigs
}

\author{
Satoshi Mikawa ${ }^{1 *}$, Shuji Sato ${ }^{1,6}$, Masahiro Nii ${ }^{2}$, Takeya Morozumi ${ }^{3}$, Gou Yoshioka ${ }^{4}$, Noriaki Imaeda ${ }^{4}$, \\ Tsuneko Yamaguchi ${ }^{5}$, Takeshi Hayashi ${ }^{1,7}$, Takashi Awata ${ }^{1}$
}

\begin{abstract}
Background: The number of vertebrae in pigs varies and is associated with body size. Wild boars have 19 vertebrae, but European commercial breeds for pork production have 20 to 23 vertebrae. We previously identified two quantitative trait loci (QTLs) for number of vertebrae on Sus scrofa chromosomes (SSC) 1 and 7, and reported that an orphan nuclear receptor, NR6A1, was located at the QTL on SSC1. At the NR6A1 locus, wild boars and Asian local breed pigs had the wild-type allele and European commercial-breed pigs had an allele associated with increased numbers of vertebrae (number-increase allele).

Results: Here, we performed a map-based study to define the other QTL, on SSC7, for which we detected genetic diversity in European commercial breeds. Haplotype analysis with microsatellite markers revealed a 41-kb conserved region within all the number-increase alleles in the present study. We also developed single nucleotide polymorphisms (SNPs) in the 450-kb region around the QTL and used them for a linkage disequilibrium analysis and an association study in 199 independent animals. Three haplotype blocks were detected, and SNPs in the 41$\mathrm{kb}$ region presented the highest associations with the number of vertebrae. This region encodes an uncharacterized hypothetical protein that is not a member of any other known gene family. Orthologs appear to exist not only in mammals but also birds and fish. This gene, which we have named vertnin (VRTN) is a candidate for the gene associated with variation in vertebral number. In pigs, the number-increase allele was expressed more abundantly than the wild-type allele in embryos. Among candidate polymorphisms, there is an insertion of a SINE element (PRE1) into the intron of the $\mathrm{Q}$ allele as well as the SNPs in the promoter region.
\end{abstract}

Conclusions: Genetic diversity of VRTN is the suspected cause of the heterogeneity of the number of vertebrae in commercial-breed pigs, so the polymorphism information should be directly useful for assessing the genetic ability of individual animals. The number-increase allele of swine VRTN was suggested to add an additional thoracic segment to the animal. Functional analysis of VRTN may provide novel findings in the areas of developmental biology.

\section{Background}

In mammals, the vertebral formula shows developmental constraint [1]. The number of cervical vertebrae is fixed at 7 , and the total number of thoracic and lumbar vertebrae tends to be 19 , although the specific counts vary among species. For example, in the Monotremata, Marsupialia, Lagomorpha, Rodentia, and Artiodactyla, the total number of thoracic and lumbar vertebrae is conserved at 19 , which is thought to be the primitive form.

\footnotetext{
* Correspondence: mikawa@affrc.go.jp

'Animal Genome Research Unit, National Institute of Agrobiological Sciences, Tsukuba, Ibaraki 305-8602, Japan

Full list of author information is available at the end of the article
}

In comparison, this number is increased in the Perissodactyla (e.g., horse, 24 vertebrae) and Carnivora (e.g., dog, 20 vertebrae) and is reduced to 17 in the Primata. However, these changes are lineage specific, and variation is restricted within each species, as is seen in the Primata [2].

Wild boars, which are the ancestors of modern domestic pigs, have 19 vertebrae. In comparison, European commercial breeds have increased numbers ranging from 20 to 23 [3]. These breeds have long been selectively bred for enlargement of body size in order to increase meat production and improve reproductive
C Biomed Central

C 2011 Mikawa et al; licensee BioMed Central Ltd. This is an Open Access article distributed under the terms of the Creative Commons Attribution License (http://creativecommons.org/licenses/by/2.0), which permits unrestricted use, distribution, and reproduction in any medium, provided the original work is properly cited. 
performance. This process has presumably increased the number of vertebrae.

In previous papers on studies of $F_{2}$ families, we reported two quantitative trait loci (QTLs) for number of vertebrae; the loci were found on Sus scrofa chromosomes (SSCs) 1 and 7 [4-6]. These two QTLs acted independently, and each had a mainly additive effect (approximately 0.55 and 0.60 per allele, respectively). In the $\mathrm{F}_{2}$ families, wild boar and Asian local breeds had the wild-type alleles at both QTLs. All the alleles of European breeds at the QTL on SSC1 increased the number of vertebrae, but only some of the alleles of European breeds at the QTL on SSC7 increased vertebral number. At the QTL on SSC1, we found a 300-kb region fixed in a variety of European commercial breeds; the gene encoding an orphan nuclear receptor/germ cell nuclear factor (NR6A1/GCNF) was located in this region [7]. The European allele of NR6A1/GCNF had a nonsynonymous substitution $(\mathrm{C} \rightarrow \mathrm{T}$ at nucleotide 748 of AB248749; Pro192Leu) that led to three times the binding activity to cofactors (NCOR1 and RAP80) as with the wild-type allele product. However, genetic variation of NR6A1/GCNF was not detected in today's commercial-breed pigs, and they have no fewer than 20 vertebrae. Interestingly, the number-increasing-type (Leu-type) alleles were also detected in some Chinese indigenous breeds, in which introgression of Western germplasm has occurred with aim of improving their productivity and the number of vertebrae has increased [8].

In the current report, we describe our map-based study of the QTL on SSC7, which was responsible for the variation in vertebral number in the today's commercial-breed pigs and may affect many phenotypic traits. Near the QTL for vertebral number on SSC7, other QTL effects for body composition [5,6], growth rate $[9,10]$, and fat deposition $[11,12]$ were also detected. We expect that our identification of the gene at this QTL will be of great benefit to the pork production industry.

\section{Results}

Defining the QTL region in a Large White population

We first developed 54 microsatellite markers in the $95 \%$ confidence interval (approximately $5 \mathrm{cM}$, or $9 \mathrm{Mb}$; Figure 1) of the QTL on SSC7 by using a comparative gene map for human and pig [13]. We also used swine genome draft sequences, Sscrofa9 published by the International Swine Genome Sequencing Consortium (SGSC; [14]) for the assignment of the markers to the swine genome (Additional file 1 Figure S1; Additional file 2 Table S1; Additional file 3 Table S2).

We next evaluated the QTL types of sires and dams in a closed breeding population of Large White (Awa-York, AY population) by using half-sib analysis of genotype

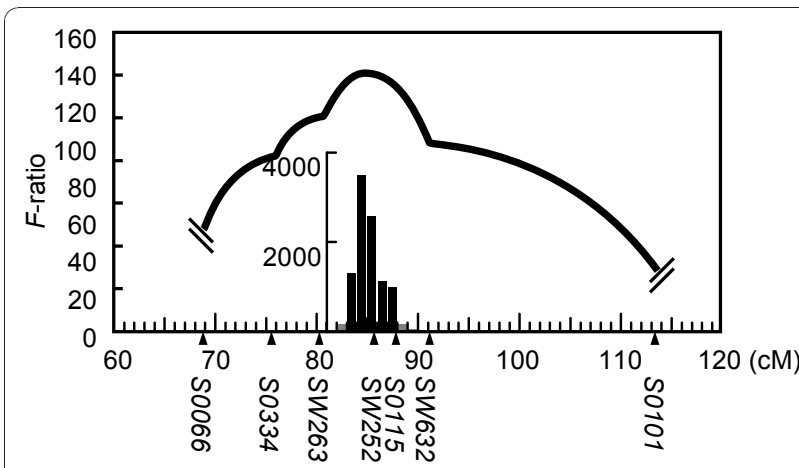

Figure 1 Interval mapping of the QTL for number of vertebrae on SSC7. A QTL analysis was performed in a JD family consisting of a Duroc sire, five Chinese Jinhua dams, six $F_{1}$ sires, $21 F_{1}$ dams, and $528 \mathrm{~F}_{2}$ progeny. Plots of the $F$-ratio for interval mapping analysis are shown. The 95\% confidence interval was analyzed with 10,000 repeats of bootstrap samples by QTL Express software and is indicated in the inset bar graphs. Black bars mean that the regions were included within $95 \%$ of samples. The $95 \%$ confidence interval for this QTL was from $83 \mathrm{cM}$ to $88 \mathrm{cM}$ on SSC7, between markers SW263 and S0115. In this analysis, a linkage map constructed by using the JD family was used.

and phenotype data for approximately 1100 progeny. We found nine heterozygotes that had the wild-type allele (wt) and the allele associated with increased numbers of vertebrae (number-increase allele, Q). We also found six homozygotes: five had the number-increase type $(\mathrm{Q} / \mathrm{Q})$ and one had the wild type (wt/wt), as judged by $Z$-score test [15] and multiple comparison test (Table 1 ). We therefore had $19 \mathrm{Q}$ alleles and $11 \mathrm{wt}$ alleles.

For these 30 alleles in 15 animals, the haplotypes of 57 microsatellite markers, including the 54 novel markers, were determined within the $95 \%$ confidence interval (Figure 2). We first searched haplotypes of some of the markers fixed within the $95 \%$ confidence interval of the $\mathrm{Q}$ allele, and found that a haplotype of two markers SJ7106 and SJ7101 was conserved (allele types 1 and 2 in Figure 2). We next searched for identical-by-descent (IBD) regions distributed in the $95 \%$ confidence interval of both the $\mathrm{Q}$ and the wt alleles. Because we considered these IBD regions to be inconsistent with the phenotypic variation, we excluded them from the candidate region. In this case, the candidate region was narrowed between $5 J 7088$ and SJ7040, and contained the two markers SJ7106 and SJ7101 described above and SJ7008 (Figure 2).

For further analysis, nine microsatellite markers (SJ7113, SJ7139, SJ7136, SJ7126, SJ7121, SJ7114, SJ7099, SJ7107, and SJ7103) were developed from swine genome draft sequences, namely BAC clones $\mathrm{CH} 242-92 \mathrm{H} 3$ and CH242-154M12, to which SJ7106, SJ7101, and SJ7008 were assigned (Additional file 2 Table S1); the subsequent analysis revealed that the haplotypes of seven 
Table 1 Half-sib analysis of QTL on SSC7 in a Large White population (AY population)

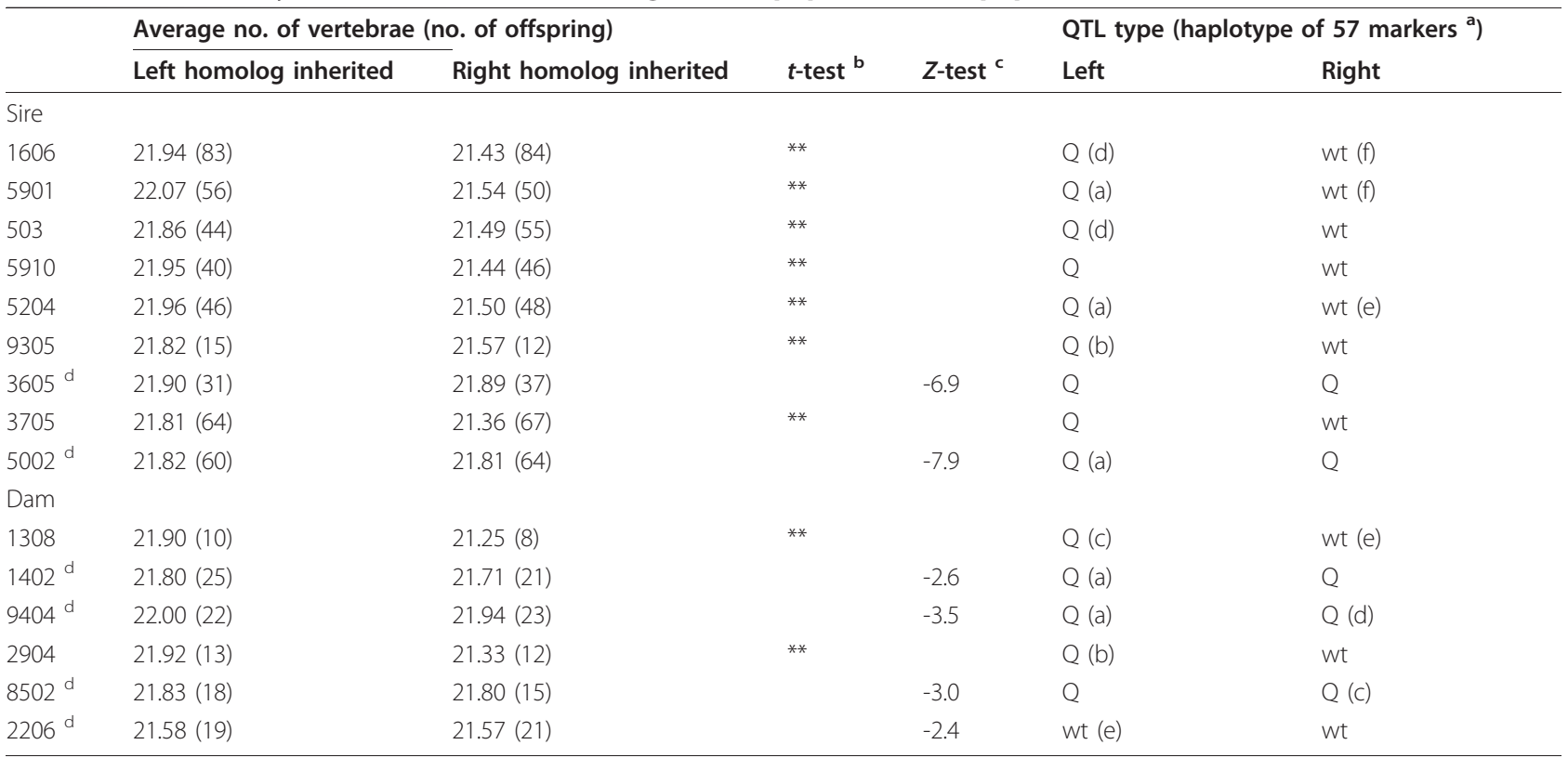

The two homologous chromosomal regions for each individual are shown arbitrarily as Left or Right. Q and wt mean the vertebral-number-increase allele and the wild-type allele, respectively.

a The same letters indicate identical haplotypes of the 57 markers within the $95 \%$ confidence interval.

${ }^{b}$ Heterozygosity was judged by $t$-test (** means $P<0.01$ ).

' Homozygosity was judged by Z-score $<-2.0$, in which the Q-to-wt substitution effect was 0.49 , as calculated with the data from nine heterozygotes in the Large White population.

${ }^{\mathrm{d}} \mathrm{QTL}$ type (Q/Q or wt/wt) was judged by multiple comparison analysis of vertebral number.

markers, from SJ7121 to SJ7103, were conserved among the $19 \mathrm{Q}$ alleles of the AY population (upper red box in Figure 3). The two markers SJ7106 and SJ7101 were located in the conserved region.

\section{Defining the QTL region by using the parental animals in $F_{2}$ families}

Haplotype analysis was performed also with the 11 European-breed pigs used as parents in the $F_{2}$ families; the QTL alleles (14 Q and eight wt alleles) of these parents were characterized in our previous study [6]. For the 14 Q alleles, the haplotypes of six markers from SJ7088 to SJ7114 were conserved (lower red box in Figure 3). Only two markers (SJ7121 and SJ7114) were common to these two sets of conserved haplotypes. The QTL was therefore judged to be located between the flanking markers SJ7126 and SJ7099, which were approximately $41 \mathrm{~kb}$ apart (Figure 4).

The corresponding region of the human genome encodes a hypothetical protein, C14orf115. Also, in pigs, parts of a transcript for the hypothetical protein have been submitted as expressed sequence tags (ESTs BE032408.1, BE032606.1, and BF198822.1) and miscRNAs (XR_045716 and XR_045719). We also cloned the corresponding cDNA (AB550854) from swine embryos by RT-PCR (Additional file 4 Figure S2).
The swine gene consisted of two exons, as in humans; the start codon, the acceptor and donor sites of the intron, and the stop codon in the second exon were identical to those in humans.

\section{Association of SNPs with number of vertebrae, and linkage disequilibrium analysis}

By using the sequences of the $\mathrm{CH} 242$ series of BAC clones of the SGSC, we analyzed the genomic structure of the 450-kb region around the QTL and constructed a provisional gene map (Figure 4; Additional file 5 Table S3). We also developed 26 single nucleotide polymorphism (SNP) markers (Figure 5; Additional file 6 Table S4) and used them for a linkage disequilibrium analysis in 199 independent meat animals produced by mating Duroc sires and $F_{1}$ (Landrace and Large White) dams. For these meat animals, the number of vertebrae was also scored. Three haplotype blocks were detected, and six SNPs (NV004, NV015, NV090, NV025, NV035, and NV062), which were highly associated with each other $\left(R^{2}>0.98\right)$, presented the highest associations with the number of vertebrae (Figure 5). These SNPs increased the number of vertebrae with an additive effect of 0.51 and a dominance effect of 0.04 (calculated from Table 2). The change in vertebral number occurred in the thoracic vertebrae; differences in the 


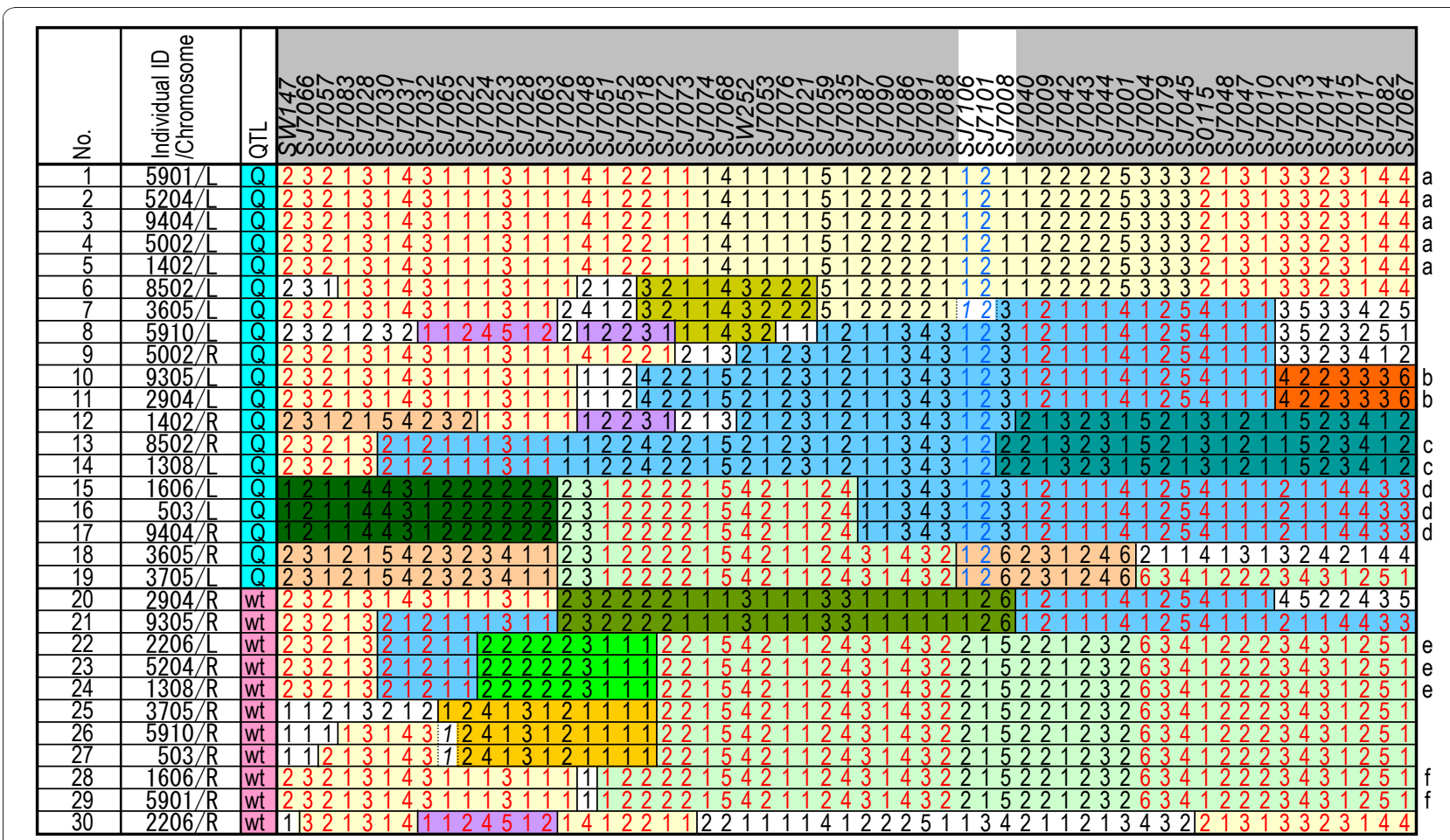

Figure 2 Haplotype analysis in the $95 \%$ confidence interval of the QTL on SSC7 in AY population. Haplotypes of microsatellite markers were analyzed in the nine sires and six dams in which allele types at the QTL (number-increase-type: Q or wild-type: wt) were determined. Microsatellite markers were genotyped with sires, dams, and their progeny, and haplotypes were reconstructed. The two homologous chromosomal regions for each individual are shown arbitrarily as Left (L) or Right (R). Numbers in each marker column represent allele types. Blocks of the same color are from the same IBD (identical-by-descent) region, which was supported by more than four markers. Italic characters indicate ambiguous alleles in determination of IBD regions. Blue letters indicate the haplotype of SJ7106 and SJ7101 conserved in the Q alleles. Red letters indicate distribution in the chromosomal regions of both the $\mathrm{Q}$ and the wt alleles. The candidate region contained three markers (SJ7106, SJ7101, and SJ7008) (white background in header row of table). Letters that are the same at the right of the panel indicate haplotypes that were identical at all 57 markers.

average number of lumbar vertebrae were not seen among the QTL types (Table 2). The six SNPs, which were between SJ7126 and SJ7099, were located from the promoter region to the second exon of the gene encoding the hypothetical proteins (Figure 4). These results strongly suggested that this hypothetical protein was responsible for the QTL on SSC7 for number of vertebrae in pigs; we named the encoding gene vertnin $(V R T N)$.

\section{Polymorphism of the QTL region}

Polymorphism analysis of the 41-kb region (AB554652) between SJ7126 and SJ7099, excluding the PRE1 sequences (swine SINE elements), revealed that only two haplotypes existed in the AY population, and these two haplotypes corresponded to the $\mathrm{Q}$ and wt alleles (Table 3). These two haplotypes consisted of 42 polymorphic sites (SNPs and indels), including the six SNPs mentioned above (Figure 4; Table 3; Additional file 6 Table S4).
We next analyzed the 42 polymorphic sites in the 11 European-breed pigs used as parents of the $F_{2}$ families (14 Q and eight wt alleles). All 14 Q alleles had the same haplotypes as that of the Q allele in the AY population. The same haplotypes (q in Table 3 ) as those in the AY population were detected in seven of eight wt alleles. In the other wt allele, which was in a Landrace sow, a unique haplotype (q' in Table 3) was detected and 33 of the polymorphic sites were identical to those of the Q allele in the AY population. These 33 were therefore excluded as candidate polymorphic sites. Of the remaining candidate polymorphic sites, five were located in the promoter region, two were in the intron, and two were in the downstream region of the gene (Figure 4). Among the nine polymorphisms, six are SNPs, two are small size of insertion or deletion, and the other is an insertion of a PRE1 element (291 bp) into the intron of the $\mathrm{Q}$ allele. These polymorphic sites of VRTN are highly related to the heterogeneity of the number of vertebrae in commercial-breed pigs, so this 


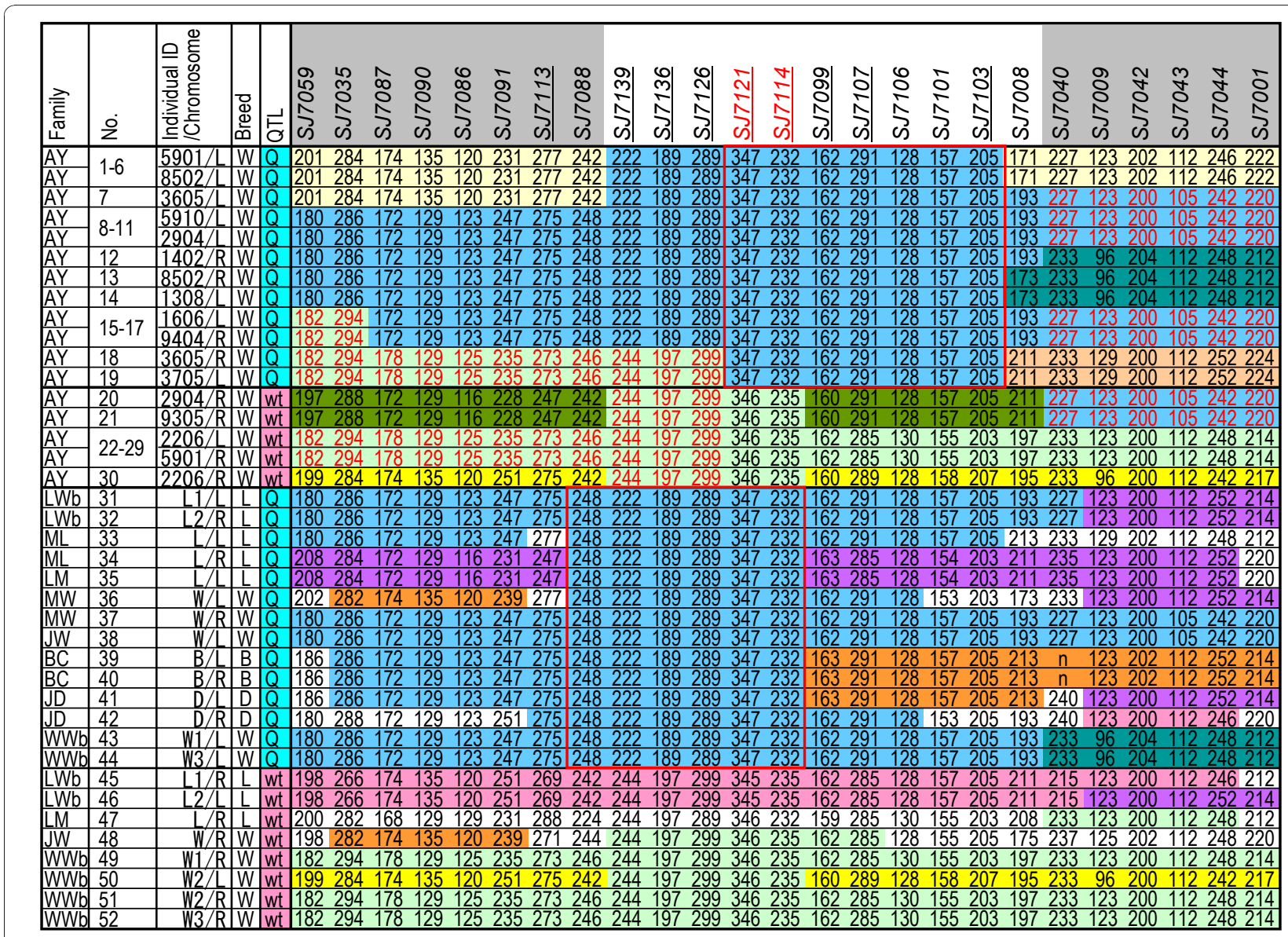

Figure 3 Detailed haplotype analysis in AY population and parent pigs of $\mathbf{F}_{\mathbf{2}}$ families. Nine novel microsatellite markers (underlined) were developed from the sequences of BAC clones, $\mathrm{CH} 242-92 \mathrm{H} 3$ and $\mathrm{CH} 242-154 \mathrm{M} 12$. These markers were added to the haplotype analysis of the AY population. In this population, some of the genetic intervals between SJ7059 and SJ7001 had identical haplotypes, and two individuals are shown for each haplotype: 5901/L and 8502/L for allele types no. 1 to no. 6; 5910/L and 2904/L for allele types no. 8 to no. 11; 1606/L and 9404/R for allele types no. 15 to no. 17; and 2206/L and 5901/R for allele types no. 22 to no. 29. Numbering is the same as in Figure 3. Haplotype analysis was also performed in the parent pigs of the $F_{2}$ families of our previous study, in which the QTL types of the parent pigs had been determined (Lower part). Numbers in each marker column are the sizes of alleles as amplified DNA fragments (in bp). Red boxes indicate conserved regions detected in the $95 \%$ confidence interval for the number-increase $(\mathrm{Q})$ allele in the AY population and in the parent pigs of the $\mathrm{F}_{2}$ families. Markers common to the two conserved regions are indicated in red letters (i.e. SJ7121 and SJ7114). The QTL was likely to be located between the flanking markers (SJ7126 and SJ7099). Breeds are: W, Large White; L, Landrace; B, Berkshire; D, Duroc. Family AY is the Awa-York population and the others are $F_{2}$ families constructed with a Japanese wild boar and two Landrace females (LWb), a Landrace male and two Meishan females (ML), a Meishan male and a Landrace female (LM), a Large White male and a Meishan female (MW), a Large White male and a Jinhua female (JW), a Clawn miniature male and a Berkshire female (BC), a Duroc male and five Jinhua females (JD), and a Japanese wild boar and three Large White females (WWb).

information will be useful for genetic diagnosis in breeding populations.

\section{Expression of VRTN gene in swine embryos}

We examined the promoter activities of the swine VRTN gene by using mouse ES cells (P19 and CGR8) but found no difference between the $\mathrm{Q}$ and wt alleles (data not shown). We detected the transcripts of mouse $V R T N$ by RT-PCR at embryonic days 6 to 10.5 (data not shown) and also tried to detect the transcripts of VRTN in swine embryos. For this analysis, we prepared heterozygous embryos by mating between $\mathrm{Q}$ and wt homozygotes, as judged by the haplotypes of $\mathrm{NVO24}$, NV107, and NV062. At five embryonic stages-days 8.0, $8.5,10.0,12.0$, and 14.0 postcoitus-embryos were collected from the uterus and RT-PCR was performed with RNA from total embryos. Amplifications were detected similarly through these periods. The PCR products were TA-cloned and SNPs were typed by a sequence-based method for each stage (Additional file 7 Figure S3). In two samples at day 8.0 , only $1.1 \%$ and $3.5 \%$ of clones were derived from the wt allele; the majority were from 


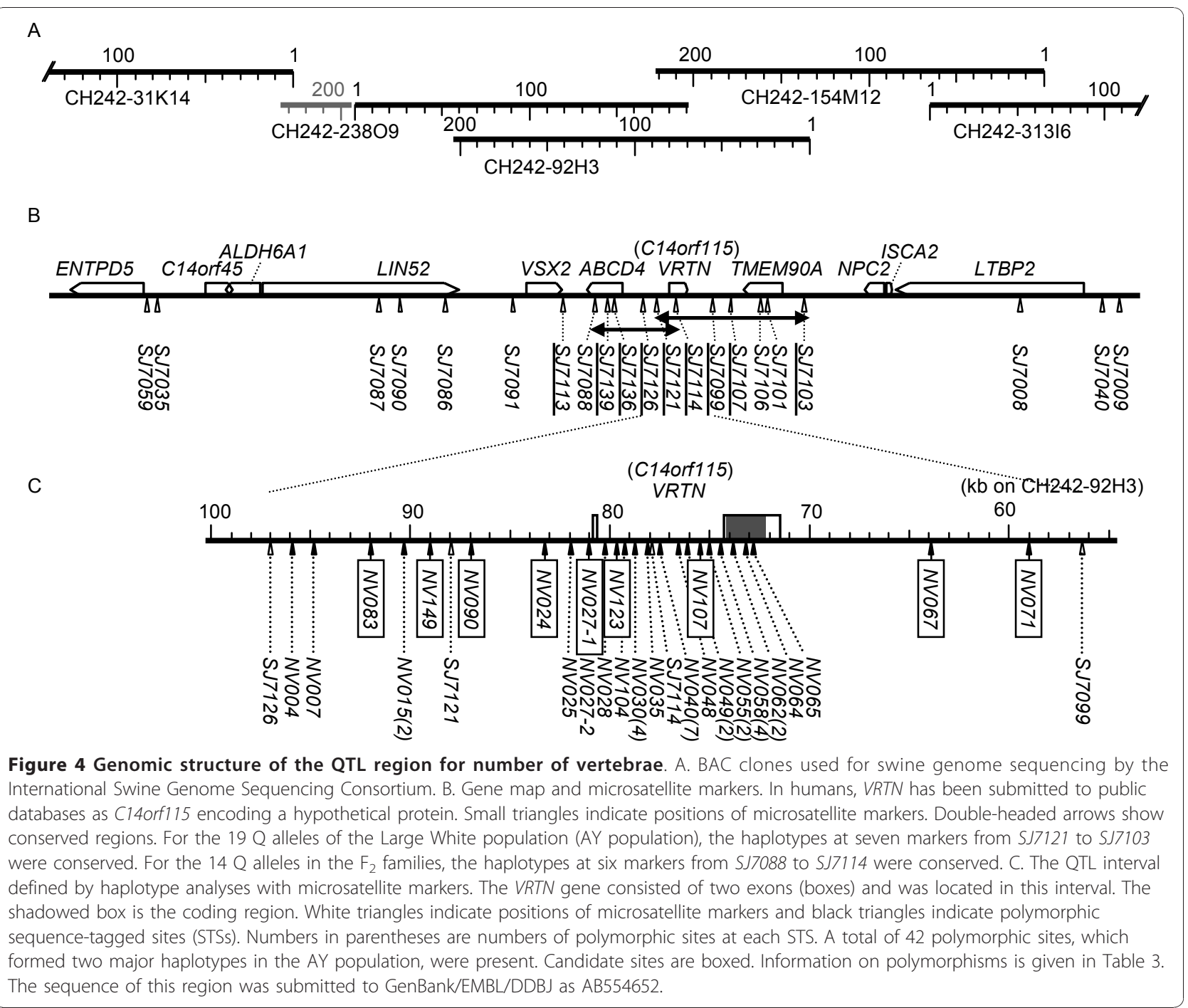

the $\mathrm{Q}$ allele (Table 4). By day 8.5, the proportion of clones from the wt allele had increased to $33.0 \%$ and $50.3 \%$, and by day 10.0 similar numbers of clones were derived from the $\mathrm{Q}$ and wt alleles. By days 12 and 14 all of the clones were from the $\mathrm{Q}$ allele. These results showed that regulation of expression of the $Q$ allele changed with the embryonic stage, and that the $\mathrm{Q}$ allele was expressed more abundantly than the wt allele.

\section{Discussion}

To map the QTL on SSC7, we used the allele type in the QTL (Q or wt), not the phenotype (vertebral number: 20, 21,22 , or 23). By using half-sib analysis with approximately 1100 progeny animals, we defined the 30 alleles of 15 parent animals. The amount of data seems small, but it is worthwhile because it is qualitative, not quantitative. These 15 animals were from a closed breeding population, the AY population. This population was derived from 10 sires and 65 dams as founder individuals and was bred for seven generations (1987 to 1993), in which average daily gain and backfat thickness were major objectives of breeding but not the number of vertebrae. After 1993, the population was maintained with 10 sires and 35 dams. We think that a large degree of recombination was accumulated in this population, making the population favorable for IBD analysis. On the other hand, unrelated individuals were used as the founders, and we expected that the $\mathrm{Q}$ alleles of some of these pigs would have common sequences in a limited region near the QTL, enabling high resolution for fine mapping similar to those used in association studies.

We started fine mapping of the QTL over a region of approximately $9 \mathrm{Mb}$ to give a $95 \%$ confidence interval in the QTL analysis. In the first step of haplotype analysis for the $19 \mathrm{Q}$ and $11 \mathrm{wt}$ alleles (Figure 2), the candidate region was fortunately narrowed to approximately $300 \mathrm{~kb}$ 


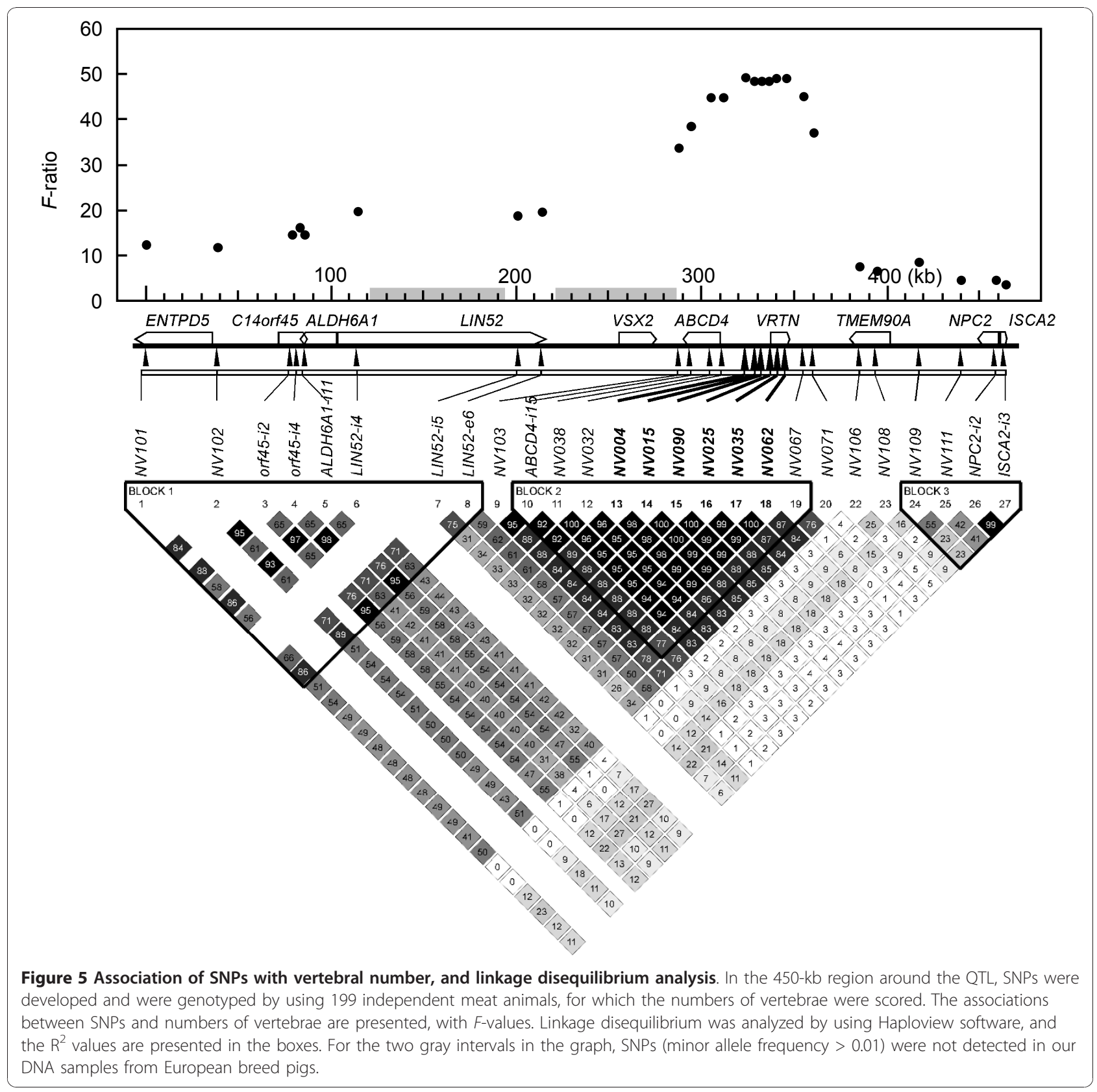

(between SJ7088 and SJ7040), but a conserved region contained only two markers in the $19 \mathrm{Q}$ alleles. The second step of the analysis, using densely located markers, revealed the conserved region with seven markers (no more than $200 \mathrm{~kb}$ ) in the Q alleles of the AY population.

In our previous study [6], we characterized the QTL alleles of parental pigs in the $F_{2}$ families, and we used these animals for further analysis. The $14 \mathrm{Q}$ alleles of these pigs (five Landrace, two Berkshire, two Duroc and five Large White) presented a conserved haplotype from $S J 7088$ to SJ7114. As a final mapping result, the candidate region for the QTL was an approximately 41-kb region between SJ7126 and SJ7099, which included the common haplotype within all the Q alleles in the present study.

The result of the fine mapping was confirmed by linkage disequilibrium analysis and an association study in independent meat animals produced with Duroc sires and $F_{1}$ (Landrace $\times$ Large White) dams. The 41-kb region was included in a haplotype block, and the SNPs developed in this region were highly associated with the number of vertebrae. We therefore concluded that the QTL was located in the 41-kb region. 
Table 2 Summary of QTL effect in meat animals

\begin{tabular}{|c|c|c|c|c|c|c|c|c|}
\hline \multirow[b]{3}{*}{ QTL } & \multicolumn{4}{|c|}{ Total number of vertebrae } & & & & \\
\hline & 20 & 21 & 22 & 23 & & & & \\
\hline & \multicolumn{4}{|c|}{ Number of individuals } & Total & \multicolumn{3}{|c|}{ Average } \\
\hline $\mathrm{Q} / \mathrm{Q}$ & & 21 & 31 & 2 & 54 & 21.65 & & \\
\hline $\mathrm{Q} / \mathrm{wt}$ & 3 & 88 & 21 & 1 & 113 & 21.18 & & \\
\hline \multirow[t]{4}{*}{$w t / w t$} & 14 & 16 & 2 & & 32 & 20.63 & & \\
\hline & 17 & 125 & 54 & 3 & 199 & 21.22 & & \\
\hline & \multicolumn{3}{|c|}{$\begin{array}{l}\text { No. of thoracic } \\
\text { vertebrae }\end{array}$} & & \multicolumn{3}{|c|}{$\begin{array}{l}\text { No. of lumbar } \\
\text { vertebrae }\end{array}$} & \\
\hline & 14 & 15 & 16 & & 5 & 6 & 7 & \\
\hline QTL & \multicolumn{3}{|c|}{ No. of individuals } & Average & \multicolumn{3}{|c|}{ No. of individuals } & Average \\
\hline $\mathrm{Q} / \mathrm{Q}$ & & 12 & 42 & 15.78 & 11 & 39 & 4 & 5.87 \\
\hline $\mathrm{Q} / \mathrm{wt}$ & 1 & 78 & 34 & 15.29 & 21 & 84 & 8 & 5.89 \\
\hline \multirow[t]{2}{*}{$w t / w t$} & 14 & 16 & 2 & 14.63 & 6 & 20 & 6 & 6.00 \\
\hline & 15 & 106 & 78 & 15.32 & 38 & 143 & 18 & 5.90 \\
\hline
\end{tabular}

QTL type was judged with NV090.

In the 41-kb region, transcripts were detected in pigs as EST in the embryonic stages. They encoded parts of a putative uncharacterized protein, which is not a member of any other known gene family. We cloned the
Table 4 Expression of VRTN alleles in heterozygous embryos of pigs

\begin{tabular}{lll}
\hline Embryonic day & $\mathbf{Q}$ & $\begin{array}{c}\text { Expression rate (\%) } \\
\text { wt }\end{array}$ \\
\hline $8.0^{\text {a }}$ sample 1 & 98.9 & 1.1 \\
sample 2 & 96.5 & 3.5 \\
$8.5^{\text {a }}$ sample 1 & 67.0 & 33.0 \\
sample 2 & 49.7 & 50.3 \\
\hline 10.0 (3 embryos) & $54.5 \pm 3.7$ & $45.5 \pm 3.7$ \\
12.0 (3 embryos) & $100.0 \pm 0.0$ & $0.0 \pm 0.0$ \\
14.0 (3 embryos) & $100.0 \pm 0.0$ & $0.0 \pm 0.0$ \\
\hline
\end{tabular}

${ }^{\mathrm{a}}$ Each sample contained five embryos.

cDNA covering the putative open reading frame from the swine embryos and we named it vertnin (VRTN) as the gene responsible for the QTL for vertebral number in pigs. A domain search of the swine homolog (C14orf115) in Ensemble (http://uswest.ensembl.org/ index.html) revealed that it had a similar motif to the helix-turn-helix domain of Transposase IS3/IS911 [16]. Although this domain is reported to be unique to bacteria, vertnin is expected to have DNA-binding activities. In our preliminary study the green fluorescent protein

Table 3 Haplotypes of polymorphic sites on number-increase-type and wild-type alleles of swine VRTN gene

\begin{tabular}{|c|c|c|c|}
\hline STS for SNP/indel ${ }^{a}$ & Q (number-increase type) & q (wild type, major) & $q^{\prime}$ (wild type, minor; found in Landrace) \\
\hline NV004 & $\mathrm{T}$ & $C$ & $T$ \\
\hline NV007 & C & G & C \\
\hline NV083 & A & $\underline{G}$ & $\underline{G}$ \\
\hline NV015(2) & $\bar{A}, \mathrm{G}$ & $C, A$ & $\mathrm{~A}, \mathrm{G}$ \\
\hline NV149 & $\underline{T}$ & $\underline{A}$ & $\underline{A}$ \\
\hline NV090 & $\underline{T}$ & $\underline{c}$ & $\underline{c}$ \\
\hline NV024 & A & $\underline{T}$ & $\underline{T}$ \\
\hline NV025 & $\bar{G}$ & A & G \\
\hline NV027(2) & $\underline{C}, C$ & $\underline{A}, T$ & $\underline{A}, \mathrm{C}$ \\
\hline NV028 & A & G & A \\
\hline NV123 & ins (PRE1 291 bp) & del (PRE1 291 bp) & del (PRE1 291 bp) \\
\hline NV104 & $\mathrm{G}$ & $A$ & $G$ \\
\hline NV030(4) & $\mathrm{A}, \mathrm{T}, \mathrm{C}, \mathrm{T}$ & $\mathrm{G}, \mathrm{G}, \mathrm{T}, \mathrm{C}$ & $\mathrm{A}, \mathrm{T}, \mathrm{C}, \mathrm{T}$ \\
\hline NV035 & C & G & C \\
\hline NV040(7) & del, A, A, G, G, C, T & $A, G, T, A, A, T, C$ & del, A, A, G, G, C, T \\
\hline NV048 & A & G & A \\
\hline NV049(2) & $\mathrm{T}, \mathrm{C}$ & $C, T$ & $\mathrm{~T}, \mathrm{C}$ \\
\hline NV107 & $\underline{\mathrm{AAA}}$ & AAAAA & $\underline{\text { AAAAA }}$ \\
\hline NV055(2) & $\mathrm{T}, \mathrm{GA}$ & C, del & T, TGA \\
\hline NV058(4) & $T, T, G, G$ & $C, C, A, C$ & $T, T, G, G$ \\
\hline NV062(2) & $C, G$ & $\mathrm{~T}, \mathrm{~A}$ & $C, G$ \\
\hline NV064 & G & A & G \\
\hline NV065 & C & $\mathrm{T}$ & C \\
\hline NV067 & del & $\underline{c}$ & $\underline{C}$ \\
\hline NV071 & $\underline{c}$ & $\underline{A}$ & $\underline{A}$ \\
\hline
\end{tabular}

The nine underlined polymorphic sites were segregated between the $Q$ and wt ( $q$ and $q^{\prime}$ ) alleles.

${ }^{a}$ Numbers in parentheses are numbers of polymorphic sites at each STS. 
(GFP)-fused vertnin protein was expressed in the nuclei of cultured cells (Additional file 8 Figure S4). The QTL on SSC7 in this study affected the number of thoracic vertebrae but not the number of lumbar vertebrae, whereas the QTL on SSC1 affected the numbers of both thoracic and lumbar vertebrae [6]. This evidence suggested that the number-increase allele of swine VRTN added an additional thoracic segment in the animal. We suspect that the expression pattern of Hox genes (e.g. Hoxa-9 or Hoxc-9, which are expressed in the terminal region of the thoracic segment and more posterior portion of mouse embryos; $[17,18]$ ) could be altered. A series of genome sequencing projects (funded by National Human Genome Research Institute; http://genome.gov/) suggested that orthologs exist not only in other mammals but also in birds and fish, and encode conserved proteins (Additional file 9 Figure S5). Vertnin is therefore likely to be an essential factor for development of the embryo in a wide range of organisms. Its functional analysis may provide novel findings in the areas of developmental biology (e.g., somitogenesis or morphogenesis), so we are planning to perform a functional analysis with model organisms such as the mouse or chicken.

Polymorphism analysis of the $41-\mathrm{kb}$ region showed that the AY population had only two haplotypes $(\mathrm{Q}$ and q), which consisted of 42 polymorphic sites. Another haplotype $\left(\mathrm{q}^{\prime}\right)$ was detected in the wt allele of a Landrace sow, in which the q' allele had no significant effect (calculated as 0.05) and the other allele (Q) increased 0.55 of vertebral number [6]. In our preliminary study it was also found in the sequence of the BAC clone L261J7 [19] (Additional file 2 Table S1). There were nine candidate polymorphic sites, and they were highly associated with each other in European commercialbreed pigs. We cannot define the causative polymorphic sites genetically, but these nine sites were associated with the number of vertebrae in commercial-breed pigs, and this information will be useful for genetic diagnosis in breeding populations.

In the swine VRTN gene, we found one nonsynonymous substitution, NV064 (G $\rightarrow$ A at nucleotide 1247 of AB550854; Gly365Asp), but it was excluded from the candidate sites because the same nucleotide $G$ was located in the Q and q' alleles (Table 3). We detected a change in the transcription of VRTN with changes in embryonic stage. It is possible that this change in expression with stage is the origin of the QTL. Among the polymorphisms of the swine VRTN gene, an insertion of a PRE1 element (one of the swine SINE elements) into the intron of the $\mathrm{Q}$ allele, along with the SNPs in the promoter region, may be a cause of the changes in expression of this allele with embryonic stage. In mouse organogenesis, expression of some genes is regulated by a mechanism that includes the activation of a SINE B2 repeat [20]. In this case, the SINE B2 acts as a boundary between the heterochromatin and euchromatin, partly by the activation of pol III and pol II RNA polymerase. In another case, SINE elements have been reported to act as enhancer elements, as is the case with the FGF8 gene [21]. In the swine VRTN gene, transcripts around the PRE1 element were detected on the $\mathrm{Q}$ allele by RT-PCR in our preliminary study (data not shown). To further explore the causative mutation as well as the biological changes induced by it, we are using molecular biological techniques to study the regulation of swine VRTN expression.

\section{Conclusions}

Genetic diversity of VRTN is the suspected cause of the heterogeneity of number of vertebrae in commercialbreed pigs associated with SSC7, so the polymorphism information should be directly useful for assessing the genetic ability of individual animals. We expect that our identification of the VRTN gene will be of great benefit to the pork production industry. The number-increase allele of swine VRTN affected the vertebral formula, especially in the thoracic segment. The functional analysis of VRTN may provide novel findings in the areas of developmental biology.

\section{Methods}

Animals

DNA samples and data for number of vertebrae came from a Large White population (named Awa-York, AY) at the Livestock Research Institute of Tokushima Prefecture in Japan. ("Awa" is the old name for Tokushima Prefecture.) This population was derived from 10 sires and 65 dams as founder individuals and was bred as a closed population for seven generations (1987 to 1993), with eight to 11 sires and 28 to 36 dams. The population was then maintained with 10 sires and 35 dams. The population in this study contained some individuals that had been used in our previous study [6].

We also used DNA samples from European-breed parent pigs of the $F_{2}$ families from our previous study [6]. These families were produced by crossing a Landrace male with two Meishan females (ML), a Japanese wild boar with two Landrace females (LWb), a Large White male with a Jinhua female (JW), a Clawn miniature [22] male with a Berkshire female (BC), a Large White male with a Meishan female (MW), a Meishan male with a Landrace female (LM), a Duroc male with five Jinhua females (JD), and a Japanese wild boar with three Large White females derived from the Awa-York population (WWb; [23]).

DNA samples from the 199 meat animals independently obtained from Chiba Prefecture, Japan, were 
prepared from the skin after slaughter; the numbers of vertebrae were also scored. These meat animals had been produced by mating Duroc sires to $F_{1}$ dams of Landrace and Large White.

\section{Scanning for QTL on SSC7 for number of vertebrae}

A QTL scan on SSC7 was performed for number of vertebrae in the Jinhua $\times$ Duroc cross population (JD family). An interval mapping based on the least-squares method developed for an outbred population [24] was used. The 95\% confidence intervals for the QTL on SSC7 were obtained by bootstrap analysis of 10,000 repetitions with QTL Express software [25,26]. In this analysis, a linkage map constructed with the JD family was used.

\section{Isolation of bacterial artificial chromosome (BAC) clones and development of microsatellite markers}

A comparative map between pig and human [13] showed that the QTL region of SSC7 corresponds to a region on human chromosome 14 . Swine sequence tagged sites (STSs) were developed from swine ESTs or swine genomic sequences, which were obtained by BLAST searches with human gene sequences (Additional file 2 Table S1). BAC clones [19] were screened with these swine STSs, and microsatellite sequences were isolated from the BAC clones by using a direct sequencing method reported previously [27]. Recently, the SGSC has made progress in swine genome sequencing [14]. The sequences of the microsatellite markers were assigned in the swine genome draft sequence, Sscrofa9 assembly as well as the BAC clones ( $\mathrm{CH} 242-$ serieas) for the sequencing.

\section{Half-sib analysis on SSC7 for number of vertebrae in a Large White population}

We scored the number of vertebrae in 1338 individuals produced from a Large White population (AY population). The numbers of vertebrae were $20(<1 \%), 21$ (33\%), $22(64 \%)$, and 23 (3\%). Using the microsatellite markers $S W 147$ (90.1 cM on the MARC map; [28]), SW252 (99.4 cM), and S0115 (102.2 cM), which were located on SSC7 near the QTL for number of vertebrae, we genotyped the 1338 progeny as well as their 19 sires and 69 dams. Haplotypes consisting of these three microsatellite markers on each homologous chromosome were reconstructed by using the genotype data of the sires, the dams, and their progeny. Among the 1338 progeny, 1122 were classified into two groups on the basis of the haplotype inherited from their sire or dam. We compared the average numbers of vertebrae in the two groups of progeny for each sire or each dam and used $t$-tests to evaluate the significance of the differences. Sires and dams were considered to be heterozygous $(\mathrm{Q} / \mathrm{wt})$ at the QTL when significant differences $(P<0.01)$ were detected. Moreover, to detect sires and dams homozygous for the QTL, we performed Ztests in accordance with the method of Nezer et al. [15]. The Q-to-wt substitution effect was set at 0.49 , which was derived from the value $0.49 \pm 0.10$ calculated from nine heterozygotes in the AY population. Sires and dams were judged to be homozygous when $Z<-2.0$. QTL types of homozygotes (Q/Q or wt/wt) were judged by Tukey's multiple comparison of the average number of vertebrae of each progeny group with those (Q: 21.92 and wt: 21.43 ) of the total groups of $Q$ and $w t$ alleles inherited by the progeny (371 and 382, respectively). QTL types of homozygotes were confirmed by comparison of the haplotypes of 57 markers in the 95\% confidence interval with those of heterozygotes (Table 1). Reconstruction of the haplotypes of the 57 markers is described below.

\section{Haplotype analysis of high-density microsatellite markers in the $95 \%$ confidence interval of the QTL for number of vertebrae on SSC7}

For the sires and dams of the AY population and for the parent pigs of the $F_{2}$ families, haplotypes of 57 microsatellite markers in the $95 \%$ confidence interval (approximately $5 \mathrm{cM}, 9 \mathrm{Mb}$ ) of the QTL on SSC7 were analyzed. Haplotypes of the markers were reconstructed by using genotype data on the sires, dams, and their progeny: samples of one sire, one dam, and six progeny were used for each full-sib family. For IBD identification, the haplotypes of their progenitor animals were determined and recombination sites were analyzed. IBD regions supported by more than four markers were shown in Figures 2 and 3.

\section{Structure analysis of the swine genomic region around the QTL for number of vertebrae}

In the QTL region on SSC7, we found some errors in the Sscrofa9 assembly. The sequence of BAC clone $\mathrm{CH} 242-92 \mathrm{H} 3$ (Figure 4) was inserted inversely between those of $\mathrm{CH} 242-238 \mathrm{O} 9$ and $\mathrm{CH} 242-154 \mathrm{M} 12$ without overlapped sequences, and some regions were duplicated (SJ7088, SJ7106, SJ7101, SJ7008 in additional file 2 Table $\mathrm{S} 1)$. Then we constructed the gene map again. With the sequences of the BAC clones CH242-31K24, CH242238O9, CH242-92H3, CH242-154M12, and $\mathrm{CH} 242-$ 313I6, BLAST searches were performed against the human genome sequence. Sequence information on the genes in the human homologous region was used for the construction of a provisional gene map (Additional file 5 Table S3). In each gene, exons were estimated with the human gene sequence or swine mRNA sequences. The gt-ag rule was confirmed for each intron. For the swine VRTN gene, RT-PCR was 
performed with three sets of primer pairs-sVRTN 1, 5' agacggtccatgctcaatg-3' and 5'-tgcagtgctccaggtacaac-3'; sVRTN 2, 5'-aggaggtggaggctgaaagt-3' and 5'-actcaggtccctgaccctct-3'; and sVRTN 3, 5'-cagcttctgttggggaaaag-3' and 5'-ctgtggggtccagaacagat-3'-in the genomic sequence corresponding to the human C14orf115 sequence (Additional file 4 Figure S2).

\section{Association of SNPs with number of vertebrae, and linkage disequilibrium analysis around the QTL region} PCR primers were designed on the basis of the sequences of the BAC clones of the SGSC, and SNPs were developed from eight samples of DNA from meat animals produced by the mating of Duroc sires and $F_{1}$ dams of Landrace and Large White (Additional file 6 Table S4; Figure 5). SNPs were genotyped for 199 independent samples from meat animals, for which the numbers of vertebrae were scored. Analysis of variance was performed for each SNP. Linkage disequilibrium was analyzed in the same 199 meat animals by using Haploview software [29]; the $\mathrm{R}^{2}$ values are shown in Figure 5.

\section{Polymorphism analysis of swine VRTN gene}

Polymorphism analysis for the approximately 41-kb region between microsatellite markers SJ7126 and SJ7099 was performed in the AY population. DNA samples from sires 3605 (Q/Q), 5002 (Q/Q), 1606 (Q/wt), and 5901 (Q/wt), and from dam 2206 (wt/wt) of the AY population (Table 1) were used. Sequences were determined by application of a series of PCR and direct sequencing methods to each sample. Polymorphic sites segregating between the $\mathrm{Q}$ and wt alleles, except for those in PRE1 elements, are shown in Figure 4 and Table 3. These polymorphic sites were elucidated by using other DNA samples from the AY population and the parent pigs of the $F_{2}$ families, for which QTL types were determined. A haplotype that we found in a Landrace female and considered to be a wt allele is also presented in Table 3.

\section{Expression analysis of VRTN in swine embryos}

Heterozygous embryos were prepared by reciprocal matings of $\mathrm{Q}$ and wt homozygotes, which were determined by the haplotypes of NV024, NV107, and NV062 in the swine VRTN gene. At five embryonic stages-days 8.0, $8.5,10.0,12.0$, and 14.0 postcoitus-embryos were collected from the uterus after slaughter. RNA was prepared with Trizol (Invitrogen) and RT-PCR was performed with PCR primers for NV062 (Additional file 7 Figure S3). Amplified DNA fragments were cloned by using a TOPO-TA cloning kit (Invitrogen), and two SNPs in NV062, which was located in the second exon, were genotyped from 96 clones in each stage by direct- sequencing of PCR fragments from the inserts. On each of days 8.0 and 8.5 , five embryos were taken together as one sample and RNA was prepared. At the other stages postcoitus, RNA was prepared separately from each embryo. At each of days 8.0 and 8.5, two samples were from reciprocal matings. At day 10, one embryo was from a cross between a wt/wt male and a Q/Q female, and two were from a reciprocal cross. At day 12, embryos were from a cross between a wt/wt male and a Q/Q female. At day 14, embryos were from a cross between a Q/Q male and a wt/wt female.

\section{Additional material}

Additional file 1: Figure S1: Development of microsatellite markers in the $95 \%$ confidence interval of the QTL on SSC7. A. A part of the gene map for the human chromosome 14 (from the human genome reference genome assembly of $\mathrm{NCBI}$, Build 37.1). Human sequences in the region corresponding to the QTL on SSC7 were used to search for homologous swine sequences by BLAST analysis. PCR primers for STSs were designed in these swine sequences. B. Swine BAC clones screened with STSs. C. Swine microsatellite markers developed in this study. Microsatellite markers were isolated from the BAC clones by a direct sequencing method using two-nucleotide repeats such as $(C A)_{10}$ for sequencing primers. D. A part of the SSC7 sequence map (from the swine genome draft sequence, Sscrofa9 assembly, published by the International Swine Genome Sequencing Consortium). The microsatellite markers developed in this study and those on a linkage map developed by Rohrer [28] were assigned to the Sscrofa9 assembly. The underlined markers have not yet been found in the Sscrofa9 assembly. Dotted lines indicate that markers were assigned to multiple positions. E. A part of the SSC7 linkage map for microsatellite markers, developed by Rohrer [28]

Additional file 2: Table S1: Development of swine STSs and microsatellite markers

Additional file 3: Table S2: Information on microsatellite markers Additional file 4: Figure S2: Swine VRTN cDNA. A swine VRTN CDNA. (number-increase-type; AB550854) was cloned by RT-PCR with three primer pairs: SVRTN 1 (red underlines), sVRTN 2 (green), and SVRTN 3 (blue). Comparison with the swine genome draft sequences revealed that exon 1 extended from the 1 st to the 152nd nucleotides and exon 2 ranged from the $153 \mathrm{rd}$ to the end. The coding region was 2,094 bp long (154-2,247) and predicted to encode a protein of 698 amino acids. The positions of SNPs in a Large White population (the AY population) are shown with blue background.

Additional file 5: Table S3: Locations of genes surrounding the QTL Additional file 6: Table S4: Polymorphic markers used in this study. Additional file 7: Figure S3: Expression analysis of VRTN in swine embryos.

Additional file 8: Figure S4: Expression of the green fluorescent protein (GFP)-fused vertnin in cultured cells. The plasmid vector pCDNA-DEST47-VRTN, which encoded GFP-fused vertnin, was constructed from VRTN cDNA and pcDNA-DEST47 plasmid vector (Invitrogen). NIH$3 \mathrm{~T} 3$ and HeLa cells $\left(1 \times 10^{4}\right.$ cells/chamber $)$ were seeded on BioCoat Poly-D-Lysine 4-well Culture Slides (BD Biosciences), and then the plasmid vectors pcDNA-DEST47-VRTN and pcDNA/GW-47/CAT (Invitrogen), which encoded a GFP-fused CAT (chloramphenicol acetyltransferase) and was a control for cytoplasmic expression, were transfected into cells by using FuGENE 6 (Roche Diagnostics). Forty-eight hours after transfection, the cells were washed and the nuclei were counterstained with 4,6-diamidino-2-phenylindole (DAPI) (Invitrogen). The cells were mounted with the anti-bleaching reagent DABCO (Invitrogen) and analyzed by fluorescence microscopy to examine green (GFP) and blue (DAPI) fluorescence. DAPI staining indicates the locations 
of nuclei, and GFP-fused vertnin has a similar expression pattern in both types of cell.

Additional file 9: Figure S5: Orthologs of swine vertnin protein. A. In the public database, protein sequences probably coded by orthologous genes of swine VRTN were found not only in mammals, including opossum, but also in a bird (zebra finch) and fish (zebrafish and tetraodon). The orthologous genes were conserved at the start and stop codons, so it seems that VRTN encodes a functional protein. Alignment was performed with ClustalW software. Underlines in the swine sequence indicate the homologous region to helix-turn-helix domain of Transposase IS3/IS911. B. The identities (\%) of amino acid sequences to swine VRTN. C. Phylogenic tree of vertnin proteins constructed with ClustalW software.

\section{Acknowledgements}

This work was supported by grants to the Animal Genome Project from the Ministry of Agriculture, Forestry, and Fisheries of Japan, and by a grant-in-aid from the Japan Racing Association.

\section{Author details}

'Animal Genome Research Unit, National Institute of Agrobiological Sciences, Tsukuba, Ibaraki 305-8602, Japan. 'Livestock Research Institute, Tokushima Agriculture, Forestry and Fisheries Technology Support Center, Kamiita, Tokushima 771-1310, Japan. ${ }^{3}$ STAFF Institute, Tsukuba, Ibaraki 305-0854, Japan. ${ }^{4}$ Gifu Prefecture Livestock Research Institute, Minokamo, Gifu 5050037, Japan. ${ }^{5}$ Chiba Prefecture Livestock Research Center, Yachimata, Chiba 289-1113, Japan. ${ }^{6}$ National Livestock Breeding Center, Odakura, Nishigo, Fukushima 961-8511, Japan. ${ }^{7}$ National Agricultural Research Center, Tsukuba, Ibaraki 305-8666, Japan.

\section{Authors' contributions}

SM designed the experiment, coordinated IBD and LD mapping, analyzed genome structure, performed association study, coordinated expression analysis of swine embryos, and wrote the manuscript. SS developed STSs, microsatellite markers and SNPS, coordinated IBD and LD mapping, and performed polymorphism analysis. MN collected samples and phenotypes for IBD analysis. TM performed expression analysis of GFP-fused proteins, and coordinate expression analysis of swine embryos. GY and NI collected and prepared the samples of swine embryos. TY collected and prepared the samples for LD mapping and association study. TH performed QTL mapping and statistical analysis. TA coordinated expression analysis of swine embryos, was involved in discussions about all of the analyses performed, and assisted in manuscript preparation. All authors reviewed the manuscript.

Received: 14 July 2010 Accepted: 14 January 2011

Published: 14 January 2011

\section{References}

1. Narita $Y$, Kuratani S: Evolution of the vertebral formulae in mammals: a perspective on developmental constraints. J Exp Zool B Mol Dev Evol 2005, 304:91-106.

2. Pilbeam D: The anthropoid postcranial axial skeleton: comments on development, variation, and evolution. J Exp Zool B Mol Dev Evol 2004, 302:241-267.

3. King JWB, Roberts RC: Carcass length in the bacon pig: its association with vertebrae numbers and prediction from radiographs of the young pig. Anim Prod 1960, 2:59-65.

4. Wada $Y$, Akita T, Awata T, Furukawa T, Sugai N, Inage $Y$, Ishii $K$, Ito $Y$, Kobayashi E, Kusumoto H, Matsumoto T, Mikawa S, Miyake M, Murase A, Shimanuki S, Sugiyama T, Uchida Y, Yanai S, Yasue H: Quantitative trait loci (QTL) analysis in a Meishan $\times$ Göttingen cross population. Anim Genet 2000, 31:376-384.

5. Sato S, Oyamada Y, Atsuji K, Nade T, Sato S, Kobayashi E, Mitsuhashi T, Nirasawa K, Komatsuda A, Saito Y, Terai S, Hayashi T, Sugimoto Y: Quantitative trait loci analysis for growth and carcass traits in a Meishan $\times$ Duroc $F_{2}$ resource population. J Anim Sci 2003, 81:2938-2949.
6. Mikawa S, Hayashi T, Nii M, Shimanuki S, Morozumi T, Awata T: Two quantitative trait loci on Sus scrofa chromosomes 1 and 7 affecting the number of vertebrae. J Anim Sci 2005, 83:2247-2254.

7. Mikawa S, Morozumi T, Shimanuki S, Hayashi T, Uenishi H, Domukai M, Okumura N, Awata T: Fine mapping of a swine quantitative trait locus for number of vertebrae and analysis of an orphan nuclear receptor, germ cell nuclear factor (NR6A1). Genome Res 2007, 17:586-593.

8. Yang G, Ren J, Zhang Z, Huang L: Genetic evidence for the introgression of Western NR6A1 haplotype into Chinese Licha breed associated with increased vertebral number. Anim Genet 2009, 40:247-250.

9. Nezer C, Moreau L, Wagenaar D, Georges M: Results of a whole genome scan targeting QTL for growth and carcass traits in a Pietran $\times$ Large White intercross. Genet Sel Evol 2002, 34:371-387.

10. Edwards DB, Ernst CW, Tempelman RJ, Rosa GJ, Raney NE, Hoge MD, Bates RO: Quantitative trait loci mapping in an F2 Duroc $\times$ Pietrain resource population: I. Growth traits. J Anim Sci 2008, 86:241-253.

11. Malek M, Dekkers JC, Lee HK, Baas TJ, Rothschild MF: A molecular genome scan analysis to identify chromosomal regions influencing economic traits in the pig. I. Growth and body composition. Mamm Genome 2001, 12:630-636

12. Liu G, Jennen DG, Tholen E, Juengst H, Kleinwächter T, Hölker M, Tesfaye D, Un G, Schreinemachers HJ, Murani E, Ponsuksili S, Kim JJ, Schellander K, Wimmers K: A genome scan reveals QTL for growth, fatness, leanness and meat quality in a Duroc-Pietrain resource population. Anim Genet 2007, 38:241-252.

13. Meyers SN, Rogatcheva MB, Larkin DM, Yerle M, Milan D, Hawken RJ, Schook LB, Beever JE: Piggy-BACing the human genome II. A highresolution, physically anchored, comparative map of the porcine autosomes. Genomics 2005, 86:739-752.

14. Schook LB, Beever JE, Rogers J, Humphray S, Archibald A, Chardon P, Milan D, Rohrer G, Eversole K: Swine Genome Sequencing Consortium (SGSC): a strategic roadmap for sequencing the pig genome. Comp Funct Genomics 2005, 6:251-255.

15. Nezer C, Collette C, Moreau L, Brouwers B, Kim JJ, Giuffra E, Buys N, Andersson L, Georges M: Haplotype sharing refines the location of an imprinted quantitative trait locus with major effect on muscle mass to a 250-kb chromosome segment containing the porcine IGF2 gene. Genetics 2003, 165:277-285.

16. Haren L, Polard P, Ton-Hoang B, Chandler M: Multiple oligomerisation domains in the IS911 transposase: a leucine zipper motif is essential for activity. J Mol Biol 1998, 283:29-41.

17. Burke AC, Nelson CE, Morgan BA, Tabin C: Hox genes and the evolution of vertebrate axial morphology. Development 1995, 121:333-346.

18. Morgan BA: Hox genes and embryonic development. Poult Sci 1997 76:96-104

19. Suzuki K, Asakawa S, lida M, Shimanuki S, Fujishima N, Hiraiwa H, Murakami Y, Shimizu N, Yasue H: Construction and evaluation of a porcine bacterial artificial chromosome library. Anim Genet 2000, 31:8-12.

20. Lunyak W, Prefontaine GG, Núñez E, Cramer T, Ju BG, Ohgi KA, Hutt K, Roy R, García-Díaz A, Zhu X, Yung Y, Montoliu L, Glass CK, Rosenfeld MG: Developmentally regulated activation of a SINE B2 repeat as a domain boundary in organogenesis. Science 2007, 317:248-251.

21. Sasaki T, Nishihara H, Hirakawa M, Fujimura K, Tanaka M, Kokubo N, KimuraYoshida C, Matsuo I, Sumiyama K, Saitou N, Shimogori T, Okada N: Possible involvement of SINEs in mammalian-specific brain formation. Proc Natl Acad Sci USA 2008, 105:4220-4225.

22. Kamimura R, Suzuki S, Nozaki S, Sakamoto H, Maruno H, Kawaida H: Branching patterns in coronary artery and ischemic areas induced by coronary arterial occlusion in the CLAWN miniature pig. Exp Anim 1996, 45:149-153.

23. Nii M, Hayashi T, Mikawa S, Tani F, Niki A, Mori N, Uchida Y, FujishimaKanaya N, Komatsu M, Awata T: Quantitative trait loci mapping for meat quality and muscle fiber traits in a Japanese wild boar $\times$ Large White intercross. J Anim Sci 2005, 83:308-315.

24. Haley CS, Knott SA, Elsen JM: Mapping quantitative trait loci in crosses between outbred lines using least squares. Genetics 1994, 136:1195-1207.

25. Visscher PM, Thompson R, Haley CS: Confidence intervals in QTL mapping by bootstrapping. Genetics 1996, 143:1013-1020.

26. Seaton G, Haley CS, Knott SA, Kearsey M, Visscher PM: QTL Express: mapping quantitative trait loci in simple and complex pedigrees. Bioinformatics 2002, 18:339-340. 
27. Fujishima-Kanaya N, Toki D, Suzuki K, Sawazaki T, Hiraiwa H, lida M, Hayashi T, Uenishi H, Wada Y, Ito Y, Awata T: Development of 50 geneassociated microsatellite markers using BAC clones and the construction of a linkage map of swine chromosome 4. Anim Genet 2003, 34:135-141.

28. Rohrer GA, Alexander LJ, Hu Z, Smith TP, Keele JW, Beattie CW: A comprehensive map of the porcine genome. Genome Res 1996, 6:371-391.

29. Barrett JC, Fry B, Maller J, Daly MJ: Haploview: analysis and visualization of LD and haplotype maps. Bioinformatics 2005, 21:263-265.

doi:10.1186/1471-2156-12-5

Cite this article as: Mikawa et al:: Identification of a second gene associated with variation in vertebral number in domestic pigs. BMC Genetics 2011 12:5.

Submit your next manuscript to BioMed Central and take full advantage of:

- Convenient online submission

- Thorough peer review

- No space constraints or color figure charges

- Immediate publication on acceptance

- Inclusion in PubMed, CAS, Scopus and Google Scholar

- Research which is freely available for redistribution

Submit your manuscript at www.biomedcentral.com/submit
() Biomed Central 